\title{
High Speed and Very Large pnCCDs for X-ray and Electron Imaging
}

\author{
H.Soltau, ${ }^{*}$ S.Bjeoumikhova, ${ }^{+}$R.Eckhardt, ${ }^{*}$ R.Hartmann, ${ }^{*}$ P.Holl, ${ }^{*}$ S.Ihle, ${ }^{*}$ M.Lang, ${ }^{*}$, N.Langhoff, \\ A.Liebel, ${ }^{* *}$ A.Niculae, ${ }^{* *}$ G.Schaller, ${ }^{++}$F.Schopper, ${ }^{++}$C.Thamm, ${ }^{*}$ A.Ziegler, ${ }^{++}$L.Strüder, ${ }^{++}$ \\ *PNSensor GmbH, Römerstr. 28, D-80803 München, Germany \\ **PNDetector GmbH, Emil-Nolde-Str.10, D-81735 München, Germany \\ ${ }^{+}$IFG GmbH, Rudower Chaussee 29/31, D - 12489 Berlin, Germany \\ ${ }^{++}$MPI Halbleiterlabor, Otto-Hahn-Ring 6, D-81739 München, Germany
}

Starting from astrophysical experiments a new class of CCDs has been developed named pnCCDs. The sensors are fabricated on $450 \mu \mathrm{m}$ thick high-resistivity silicon in a double sided ultrapure process following a so-called pnDesign and -Technology. They are very radiation hard due to the fact they are not using active MOS structures in the CCD area. The CCDs are operated in a fully depleted back-side illuminated mode with a homogenous radiation entrance window adapted to the specific properties of the radiation. For X-rays the quantum efficiency between $100 \mathrm{eV}$ and $15 \mathrm{keV}$ is very high. Electrons between $1 \mathrm{keV}$ and $300 \mathrm{keV}$ can be analyzed. The pixel size varies between $36 \mathrm{um}^{2}$ and $150 \mathrm{um}^{2}$ in dependence of the application. All CCDs are operated in parallel read out providing a high read out speed. Each channel is equipped with a first amplification step namely a n-channel JFET. The amplified signal is transferred to the so-called CAMEX chip which is responsible for additional amplification, shaping and multiplexing. The noise level of the imager is very low with 2.5 ENC only. Figure 1 shows in a principal scheme the CCD working principle.

Recently we have been working intensively on two different types of pnCCD systems: on the one side a prototype camera of a high speed pnCCD with $48 \mu \mathrm{m}$ pixel size and an active area of $2 \mathrm{~cm}^{2}$ and on the other side a very large pnCCDs with a pixel size of $75 \mu \mathrm{m}$ and an overall area of $60 \mathrm{~cm}^{2}$. While the first pnCCD is dedicated to X-ray Fluorescence Analysis or Direct Electron Imaging the second type has been foreseen as X-ray Imager at the new Free Electron Laser Facilities. Figure 2 and 3 show for both pnCCD systems the silicon sensors as well as the integration into the camera.

For the high speed application the pnCCD has been divided up into a split frame and image store area. After the image has been taken the signal electrons are rapidly transferred to the two frame store areas in $30 \mu \mathrm{s}$ only. The next image is already taken while the frame store areas are read out providing a throughput of 400 images per second or (in a special read out mode) even 1000 images per second. Though a pixel size of $48 \mu \mathrm{m}$ seems to be rather large a spatial resolution of $2 \mu \mathrm{m}$ is provided using the center of mass method. A detailed view into the specifications of the CCD will be provided as well as results from experiments at a X-ray Microfocus Tube where images are taken by means of a polycapillary optic and from measurements at a Transmission Electron Microscope.

The very large pnCCD is fabricated each on a single wafer. It is read out to both sides as the smaller type but as a trigger is provided at the electron laser a frame store area is not necessary - the pnCCD is read out continuously with a frame rate up to 120 frames per second. In the current set-up both halves of the sensors can be moved to either side in dependence of the experiment requirements leaving a narrow hole of $2.4 \mathrm{~mm}$ for the X-ray beam in the closest position. Exciting images have been taken during the first experiments at the very first high energy FEL running worldwide at LCLS at SLAC close to Stanford (USA) and will be reported. 
FIG.1. Principle working scheme of a pnCCD.

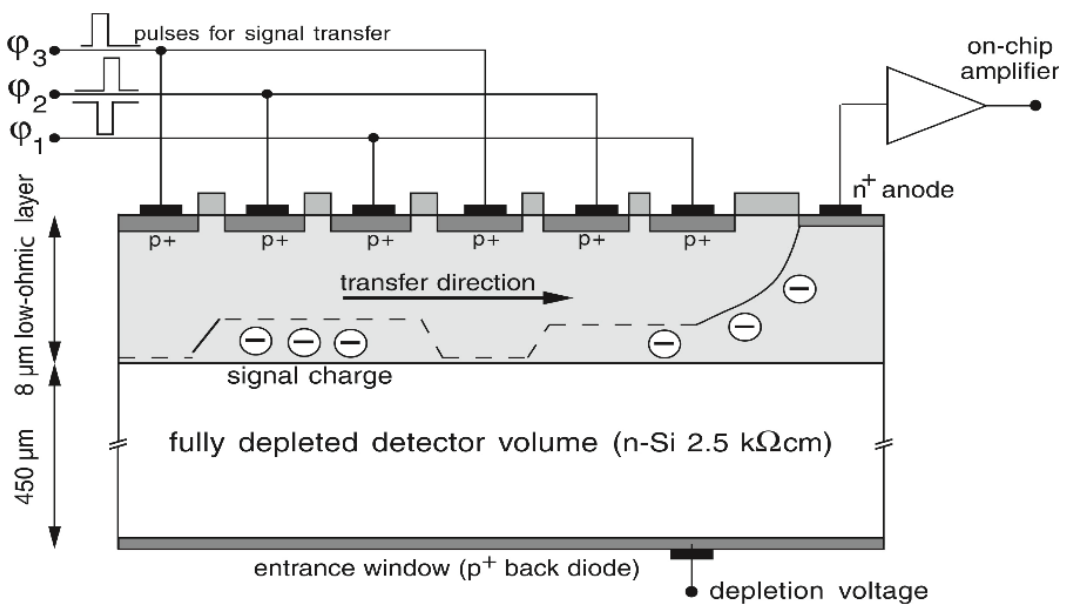

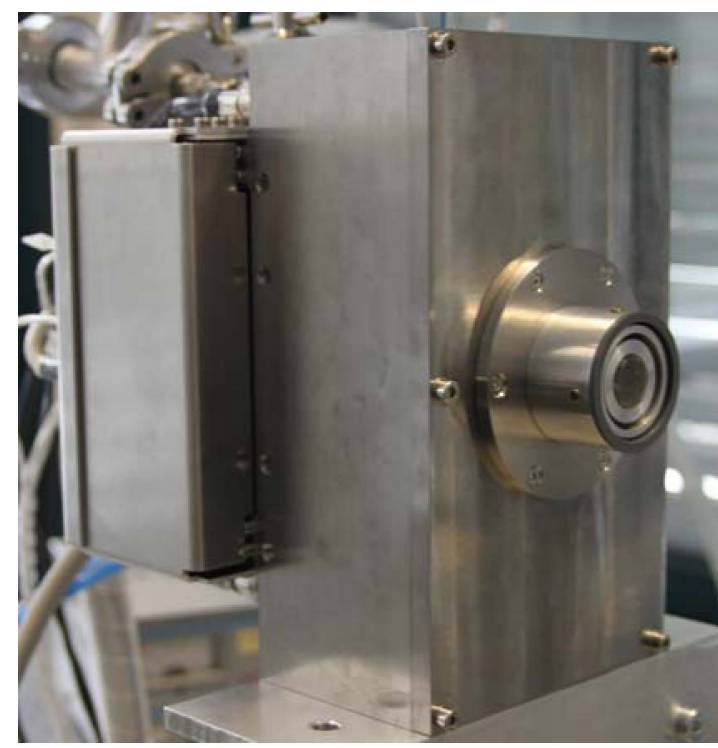

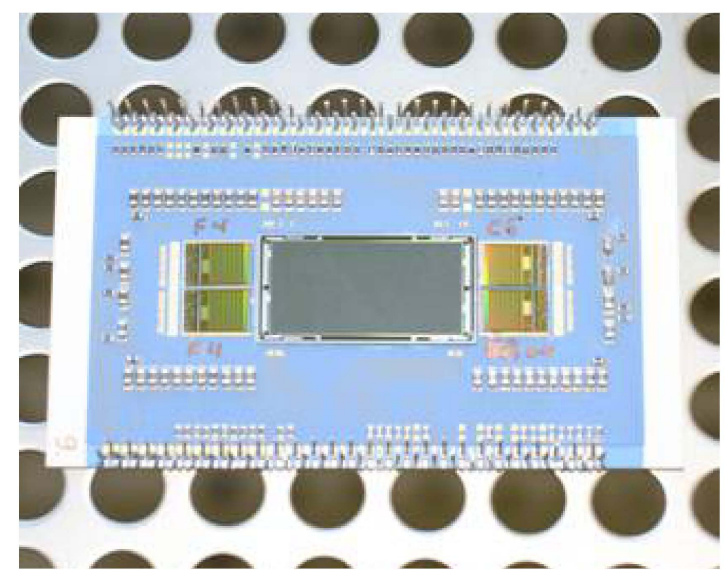

FIG.2. A high speed split frame pnCCD suitable for spectroscopic X-ray Imaging or Direct Electron Imaging in a TEM has been developed and integrated into a compact camera for test and analysis.
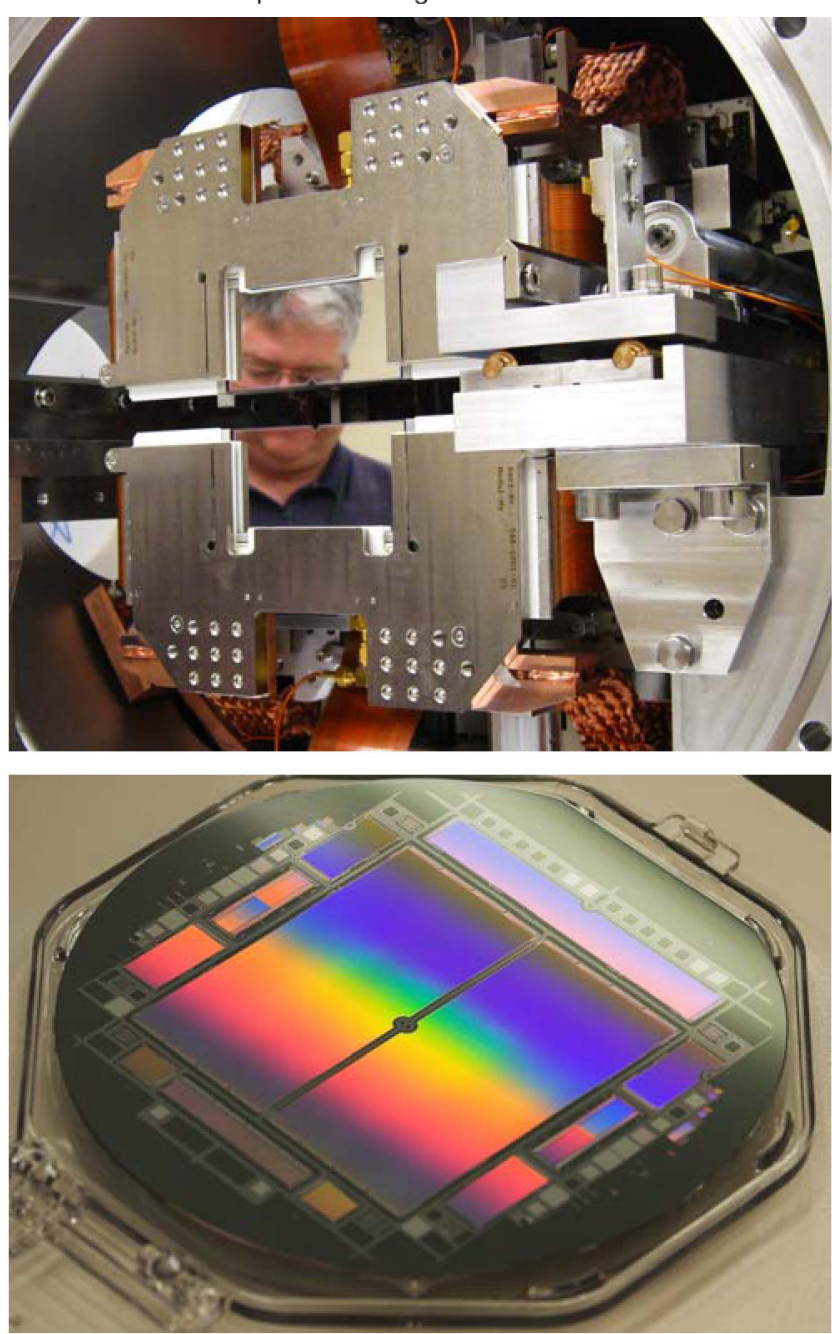

FIG 3: A very large pnCCD with $60 \mathrm{~cm}^{2}$ active area was developed as X-ray imager for the coherent X-ray sources at the new Free Electron Laser Facilities. First images have been taken at LCLS at SLAC. 